\title{
Conversation in Isolation: Australian health students' response to the COVID-19 pandemic
}

\author{
Sue Liu, Angelina Koh, Vineeta Giri, Fei Chan, Bowen Xia, Sophie He, Gayathri Bimal and Georgia Ngo \\ Conversation in Isolation Inc., Melbourne, Australia
}

\section{Introduction}

There is growing evidence for the social and psychological impact of the coronavirus disease 2019 (COVID-19) pandemic. A survey found that $28 \%$ of women and $16 \%$ of men felt lonely due to the pandemic [1], and another found that $24 \%$ of young individuals felt isolated and did not know where to turn for support [2]. Conversation in Isolation (CII) was created by Monash University medical students in 2020 to alleviate the effects of social isolation stemming from lockdown. These students were taken off clinical placements but wanted to proactively support those most vulnerable in the community in a safe but effective way. The idea of a telephone program was developed as it allowed for social distancing but also met the general need CII felt was difficult to support at the time; loneliness. Other counselling phone services such as Lifeline have experienced dramatic surges in calls as a result of people feeling anxious and isolated [3]. These services are intended to serve as one-off interventions [4], while CII serves as a longer-term intervention to re-introduce stability. This initiative has now expanded nationwide, engaging community members and volunteers studying health disciplines at tertiary institutions across Australia.

\section{Operations of $\mathrm{Cll}$}

The program involves health student volunteers being matched to community members who have signed up or been referred by peers or health professionals. Community members can express preferences regarding their volunteer, such as their spoken language, gender, and cultural background. CII endeavors to match them according to preferences to facilitate strong connections. The frequency and duration of calls are at the discretion of the matches but typically last for half an hour weekly. For community members who are unable to effectively engage via phone calls, CII also facilitates video calls, emails, text messages, and letter-writing. The CII initiative is run by a committee of 15-20 volunteers who coordinate the daily operations, support existing
Received: November 8, 2021 • Revised: November 12, $2021 \bullet$ Accepted: November 12, 2021 Corresponding Author: Georgia Ngo (https://orcid.org/0000-0002-6814-7098) Conversation in Isolation Inc., Unit 1, 92-96 Albert Street, Brunswick East, Melbourne, Australia Tel: +61.492.837.888 email: conversationinisolation@gmail.com
Korean J Med Educ 2021 Dec; 33(4): 435-439 https://doi.org/10.3946/kjme.2021.211 eISSN: 2005-7288

(C) The Korean Society of Medical Education. All rights reserved. This is an open-access article distributed under the terms of the Creative Commons Attribution Non-Commercial License (http:// creativecommons.org/licenses/by-nc/3.0/), which permits unrestricted non-commercial use, distribution, and reproduction in any medium, provided the original work is properly cited. 
participants, reach out to potential partnerships, and shape the vision of CII's future.

\section{Volunteers}

All health students studying at tertiary Australian educational institutions are eligible to volunteer. The volunteer base began as medical students from Monash University but has quickly grown to include nursing, paramedicine, counseling, and physiotherapy students, to name a few.

CII primarily recruits volunteers through social media promotion and within universities. Volunteer recruitment has been relatively easy from the impressive enthusiasm of health students to help. As a result, there are consistently more volunteers than community members which minimizes the delay between creating matches.

Volunteers are required to have a valid police check and undergo online training by CII prior to engaging with community members. During training, volunteers receive guidance on initiating their first conversation and navigating challenges that may arise. They are equipped with resources including a referral and protocol guide, a list of conversation topics, and training materials. Volunteers are also educated to recognize when their community member's mental health is out of their scope and how to escalate if they are concerned.

Furthermore, volunteers are invited to attend regular reflective debriefing sessions with Social Health Australia (SHA), an organization that focuses on the art of meaningful companioning, with which CII has partnered. In addition, CII gathers volunteer feedback via regular phone and email communication to improve the service and support volunteers who may be struggling.

\section{Community members}

Community members are individuals who have signed up to receive calls. CII's community members range from 18 to 98 years old, with a mean age of 60.5 years. The majority of sign-ups and referrals are from aged care homes and healthcare professionals such as general practitioners and social workers. Other members may learn of CII via newspaper, radio, and social media. Community members are regularly surveyed to provide feedback which supports individual community members and guides the future direction of the program.

\section{Reception and response from com- munity members and volunteers}

Eighty four point two percent of community members stated their initial motivation for signing up was to receive "friendly conversation" and $26.3 \%$ specifically stated they wanted to address their "social isolation." According to survey results, CII has successfully met these needs. CII has also received excellent testimonials from community members. One community member has said that the phone calls "gives [them] something to look forward to." Others have said it's a "wonderful way to break up the monotony" and it's "like having an opportunity to go out during the week." Overall, community reception has been extremely positive, with many participants having developed long-term friendships with their volunteers.

Similarly, volunteers have also had positive experiences and benefitted from volunteering with CII. In addition to opportunities in publication, media engagement and research, volunteers also have the opportunity to practice skills such as empathetic listening, effective 
communication, and helping others to deal with grief, which are all transferable and beneficial in their roles as future healthcare professionals. One volunteer has stated that volunteering with $\mathrm{CII}$ has been 'an enriching and rewarding experience' and that their conversations "benefitted [them] just as much or even more than the community member" allowing them to "openly talk about mental health and the impact of isolation."

Volunteering during the pandemic has been associated with positive outcomes such as the development of soft skills and improved mental health [5]. Australians who stopped volunteering in COVID-19 have been found to have a greater loss of life satisfaction compared to those who continued volunteering, and far more likely to feel lonely [6]. The program has also received positive marketing support from media enterprises, such as national newspaper 'The Age' and youth health service 'Headspace Australia.'

\section{Benefits to Australian healthcare system}

CII provides an alternative referral for doctors to help manage social isolation. During the pandemic, mental health services in the clinical and community capacity were oversubscribed [3] and it was not guaranteed that individuals would be seen in a timely manner. Support groups targeted at socially isolated communities were put on hold, further exacerbating their isolation. CII aims to fill in this gap and reduce the burden on these services.

\section{Comparison to other COVID-19 ini- tiatives}

Medical students all over the globe have risen to the occasion to aid in the pandemic. These have been in clinical support, such as student run clinics or in hospitals, particularly when there is a healthcare worker shortage [5,7-9]. These are a unique education opportunity allowing for the ongoing development of clinical skills, which is especially important when clinical placements have been disrupted. There are also community-directed initiatives such as assisting in household support for healthcare workers and elderly or immunocompromised patients [10]. The feasibility of these initiatives depends on institutional assistance from medical schools and hospitals and local restrictions.

CII was developed to navigate around the evolving restrictions in Australia. Phone calls were often the only available method of social interaction permitted between separate households; thus, the program would not be affected by restriction changes.

\section{Organizational longevity and future directions}

CII's operations are fully carried out by student volunteers in providing a service to the community free of charge. Since a large portion of the program runs with free volunteer hours, CII foresees the initiative being able to continue with a minimal budget after its inception. Beyond this, CII has been actively establishing collaborative relationships with multiple portfolios. Alongside regular reflective practice sessions for volunteers, SHA has also provided administrative and start-up support to CII.

In mid-2021, CII was successful in receiving a community grant from the local City of Monash to run a program tailored to the needs of socially isolated youth aged 18 to 25 . The pilot program will bring together over 100 young people to engage in activities focused on building social connection and mental resilience. Pro- 
gram design will be informed by industry experts and outcome evaluation will be conducted to maximize the scalability and reproducibility of positive outcomes.

CII is currently registering as an incorporated association in order to formalize our purpose and further facilitate funding and organizational activities. In the near future, CII endeavors to collaborate with other organizations and expand its outreach to culturally and linguistically diverse communities and younger demographics. As the number of COVID-19 cases in Australia decreases, CII also hopes to offer in-person interactions between volunteers and community members in line with governmental regulations. Loneliness and social isolation were significant social issues prior to the COVID-19 pandemic and will continue to have serious impacts post-pandemic. Hence, there is an ongoing and great need for CII to continue its activities and mitigate the impacts of social isolation well into the future.

\section{Conclusion}

Social isolation and loneliness have been a longstanding problem in society, which was brought to light by the COVID-19 pandemic. CII aims to sustain its operations beyond the global pandemic by reaching out to various isolated groups, collaborating with similarminded organizations, and coordinating evidence-based community programs. CII exists so that Australians do not have to go through hardships alone. It is a program affording benefits to community members and health student volunteers alike. And as long as loneliness and social isolation pull people apart, CII will continue to bring people together, one phone call at a time.

\section{ORCID:}

Sue Liu: https://orcid.org/0000-0001-7659-7008;

Angelina Koh: https://orcid.org/0000-0001-5318-4163;

Vineeta Giri: https://orcid.org/0000-0002-0750-8507;

Fei Chan: https://orcid.org/0000-0002-0870-661X;

Bowen Xia: https://orcid.org/0000-0002-0180-6159;

Sophie He: https://orcid.org/0000-0003-2199-422X;

Gayathri Bimal: https://orcid.org/0000-0002-4675-1788;

Georgia Ngo: https://orcid.org/0000-0002-6814-7098

Acknowledgements: The authors would like to acknowledge Social Health Australia and the Faculty of Medicine, Nursing and Health Sciences at Monash University for their ongoing support of this initiative.

Funding: No funding was obtained for this study.

Conflicts of interest: No potential conflict of interest relevant to this article was reported.

Author contributions: Research and referencing: SL; writing of abstract: AK, SL; writing of article: SL, AK, VG, FC, BX, SH, GB, GN; editing: BX, SH, GB, GN.

\section{References}

1. Australian Bureau of Statistics. Household impacts of COVID-19 survey: insights into the prevalence and nature of impacts from COVID-19 on households in Australia. https://www.abs.gov.au/ausstats/abs\%40.nsf/media releasesbyCatalogue/DB259787916733E4CA25855B000 3B21C?OpenDocument. Published July 2021. Accessed August 4, 2021.

2. Marlay B, Attenborough J, Kutcher V. "Living in limbo": the views and experiences of young people in Australia at the start of the COVID-19 pandemic and national response. https://www.unicef.org.au/Upload/UNICEF/Media/ Documents/UNICEF-COVID-19-Living-in-Limbo-2020. pdf. Published May 2020. Accessed August 4, 2021. 
3. Neal M. Good Friday was Lifeline's busiest day ever as coronavirus puts strain on mental health. https://www. abc.net.au/news/2020-04-19/good-friday-was-lifeline-busi est-day-ever-coronavirus-anxiety/12161 104. Published April 2020. Accessed August 4, 2021.

4. Pirkis J, Middleton A, Bassilios B, et al. Frequent callers to Lifeline. Melbourne, Australia: University of Melbourne; 2015.

5. Domaradzki J, Walkowiak D. Medical students' voluntary service during the COVID-19 pandemic in Poland. Front Public Health. 2021;9:618608.

6. Biddle N, Gray M. Volunteering during the first year of the COVID-19 pandemic (April 2021). https://csrm.cass. anu.edu.au/sites/default/files/docs/2021/5/Volunteering_d uring_the_COVID-19_pandemic_-_April_2021.pdf. Published May 2021. Accessed August 4, 2021.

7. Kratochvil TJ, Khazanchi R, Sass RM, Caverzagie KJ.
Aligning student-led initiatives and Incident Command System resources in a pandemic. Med Educ. 2020; 54(12):1183-1184.

8. Drexler R, Fröschle F, Predel C, et al. Establishing a student-run free clinic in a major city in Northern Europe: a l-year experience from Hamburg, Germany. J Public Health. 2020;42(4):793-798.

9. Bautista CA, Huang I, Stebbins M, et al. Development of an interprofessional rotation for pharmacy and medical students to perform telehealth outreach to vulnerable patients in the COVID-19 pandemic. I Interprof Care. 2020;34(5):694-697.

10. Soled D, Goel S, Barry D, et al. Medical student mobilization during a crisis: lessons from a COVID-19 medical student response team. Acad Med. 2020;95(9): 1384-1387. 